Supplementary information for:

\title{
Optimizing Cell-Free Biosensors to Monitor Enzymatic Production
}

\author{
Amir Pandi ${ }^{1}$, loana Grigoras ${ }^{2}$, Olivier Borkowski ${ }^{2 *}$, and Jean-Loup Faulon ${ }^{1,2,3}$ * \\ ${ }^{1}$ Micalis Institute, INRA, AgroParisTech, Université Paris-Saclay, Jouy-en-Josas, France \\ 2 iSSB Laboratory, Génomique Métabolique, Genoscope, Institut François Jacob, CEA, CNRS, \\ Univ Evry, Université Paris-Saclay, 91057 Evry, France \\ ${ }^{3}$ SYNBIOCHEM Center, School of Chemistry, University of Manchester, Manchester, UK \\ *To whom correspondence should be addressed: jean-loup.faulon@inra.fr or \\ olivier.borkowski@univ-evry.fr
}




\section{The supporting information consists of:}

Supplementary Figure S1. The behavior of the cell-free reaction with $10 \mathrm{nM}$ reporter DNA at different concentration of Psicose and absence of the TF.

Supplementary Figure S2. Kinetic data of the fluorescence level for the maximum fold change point at Figure 1.b.

Supplementary Figure S3. Fluorescence level for TF-doped extract with and without psicose. (matches with Figure 1.d)

Supplementary Figure S4. Fluorescence level for preincubation experiments with and without psicose (matches with Figure 1.f).

Supplementary Figure S5. Fluorescence level for 8 hours preincubation followed by adding extra fresh extract (matches with Figure 1.h).

Supplementary Figure S6. Dose-response curves for $1.5 \mathrm{~h}$ preincubation experiments.

Supplementary Table S1. List of sequences used in this study. 


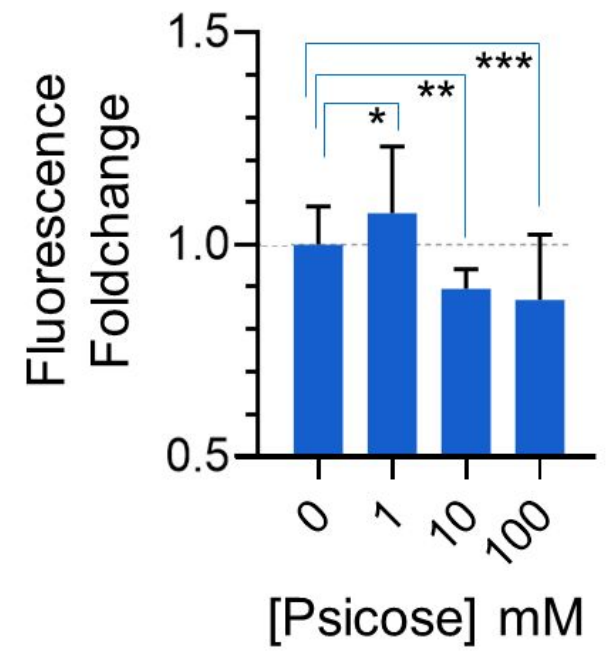

Supplementary Figure S1. The behavior of the cell-free reaction with $10 \mathrm{nM}$ reporter DNA at different concentration of Psicose and absence of the TF. This concentration of the inducer does not statistically affect the production of the reporter (The t-test p-values with fluorescence at $0 \mathrm{mM}$ vs other psicose concentrations are $0.87^{*} 0.72^{\star *}$, and $0.58^{\star \star *}$ ) 
Psicose $0 \mathrm{mM}$

- Psicose $100 \mathrm{mM}$

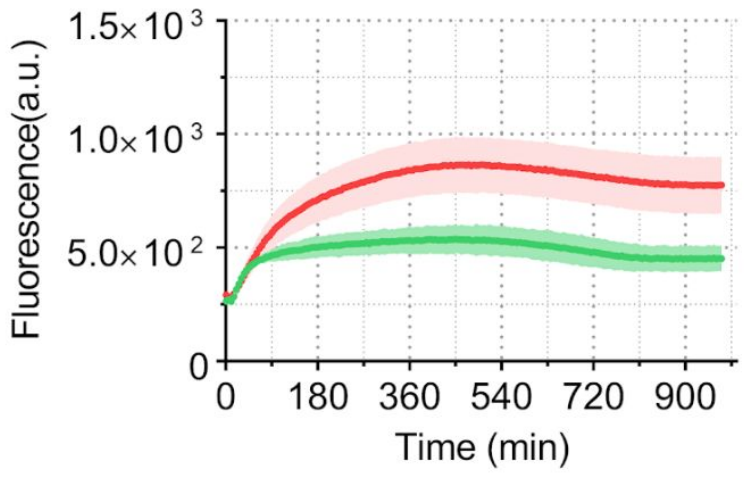

Supplementary Figure S2. Kinetic data of the fluorescence level for the maximum fold change point at Figure 1.b. The fluorescence fold change plotted in the Fe 1.b and $10 \mathrm{nM}$ reporter DNA of the $d$ bar plot in Figure 1.f is the ratio between two values at 8 hours (480 minutes) presented in this figure. The curves and shaded areas are the mean and standard deviation of three measurements from three independent reactions done in the same day using the same lysate and maxiprepped plasmids (every five-minute measurement in a plate reader). 


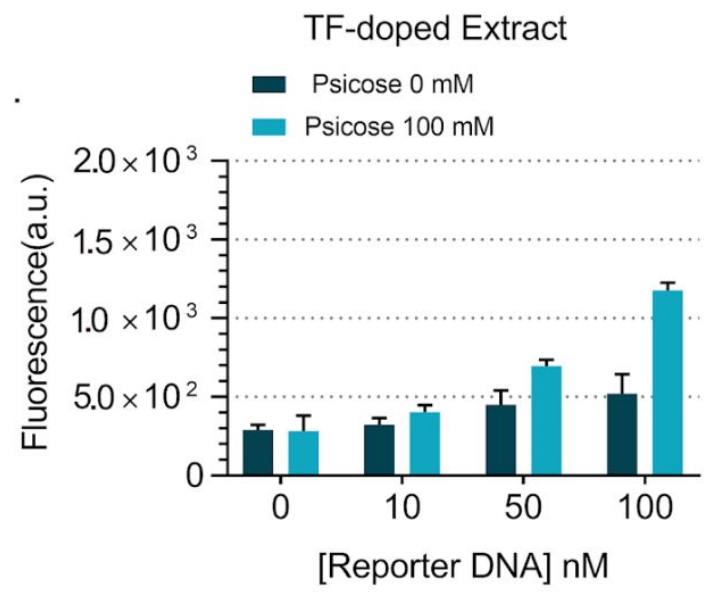

Supplementary Figure S3. Fluorescence level for TF-doped extract with and without psicose (matches with Figure 1.d). The fluorescence fold change plotted in Figure 1.d is the ratio between two bars presented in this figure for different concentrations of reporter DNA. The data and error bars are the mean and standard deviation of three measurements from three independent reactions done in the same day using the same lysate and maxiprepped plasmids. 


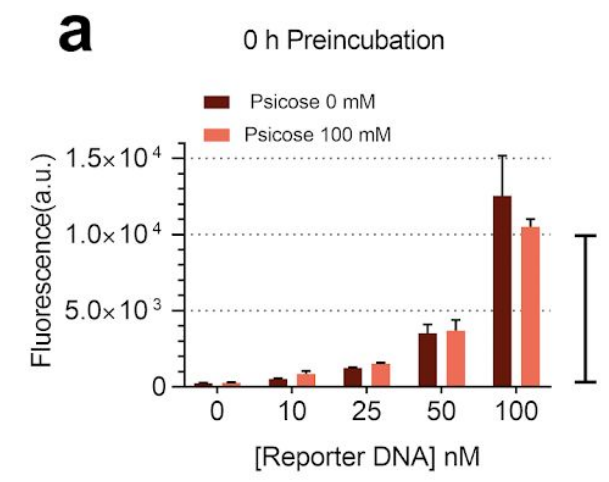

b

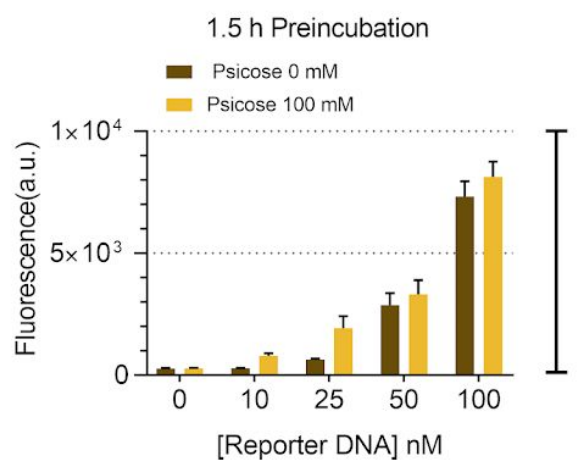

d

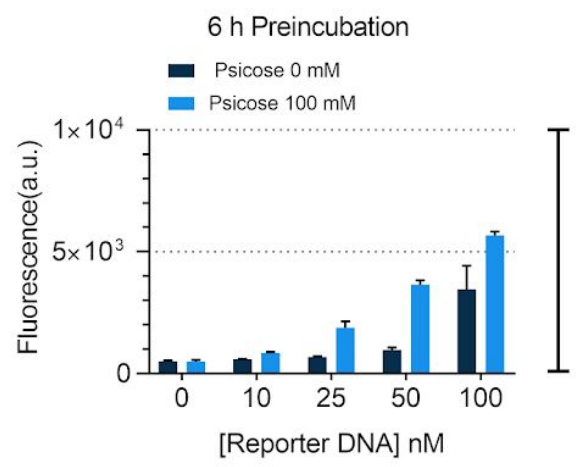

C

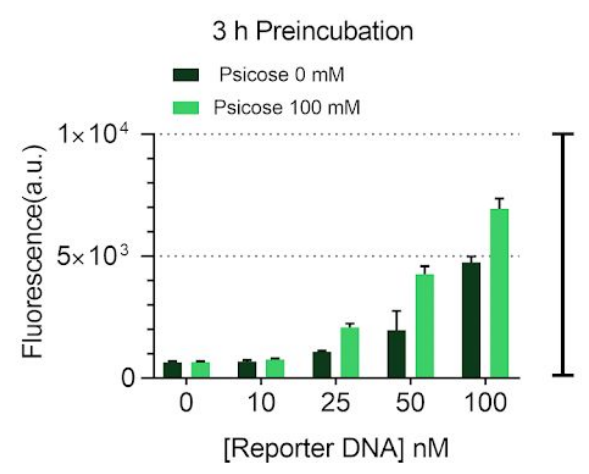

e

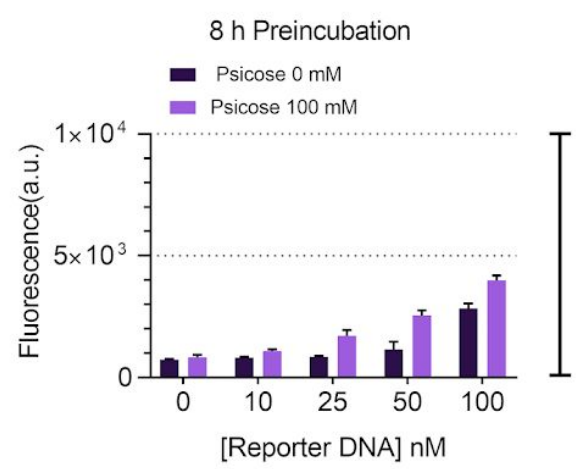


Supplementary Figure S4. Fluorescence level for preincubation experiments with and without psicose (matches with Figure 1.f). The fluorescence fold change plotted in Figure 1.f is the ratio between two bars presented in this figure for different concentrations of reporter DNA, (a) no preincubation, (b) $1.5 \mathrm{~h}$ preincubation, (c) $3 \mathrm{~h}$ preincubation, (d) $6 \mathrm{~h}$ preincubation, and (e) $8 \mathrm{~h}$ preincubation. The data and error bars are the mean and standard deviation of three measurements from three independent reactions done in the same day using the same lysate and maxiprepped plasmids. Note that the fluorescence in panel (a) is higher than other panels. Thus, panel (a) exhibits a different y-axis scale. We emphasized the difference in panel (a) scale by adding a ladder ( $\min$ and max values of all the other $y$-axis scale) at the right side of every graph. 
a

1:1 Fresh Extract

- Psicose $0 \mathrm{mM}$

- Psicose 100 mM

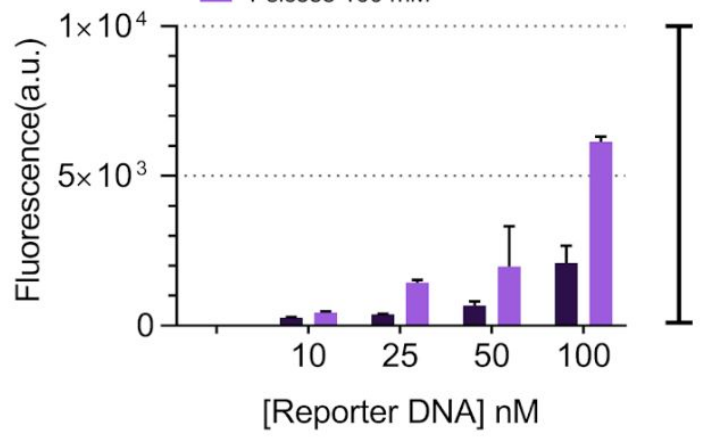

b 1:2 Fresh Extract

Psicose $0 \mathrm{mM}$ - Psicose $100 \mathrm{mM}$

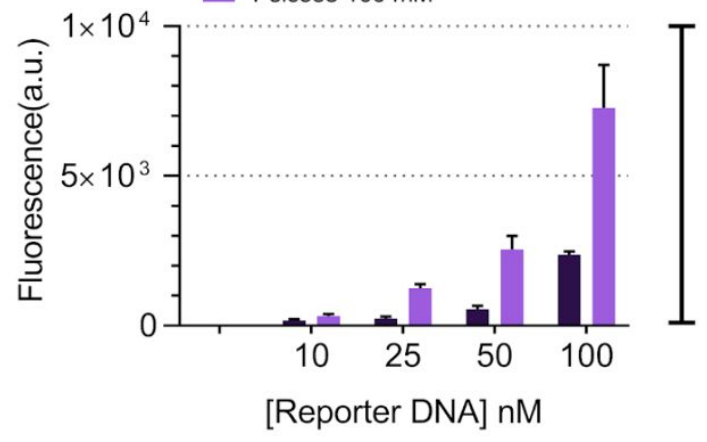

Supplementary Figure S5. Fluorescence level for 8 hours preincubation followed by adding extra fresh extract (matches with Figure 1.h). The fluorescence fold change plotted in Figure 1.h is the ratio between two bars presented in this figure for different concentrations of reporter DNA, (a) adding the same amount of the fresh extract as the preincubated reaction (15 $\mu \mathrm{l})$, and (b) adding twice more of the fresh extract $(30 \mu \mathrm{l})$. The data and error bars are the mean and standard deviation of three measurements from three independent reactions done in the same day using the same lysate and maxiprepped plasmids. 


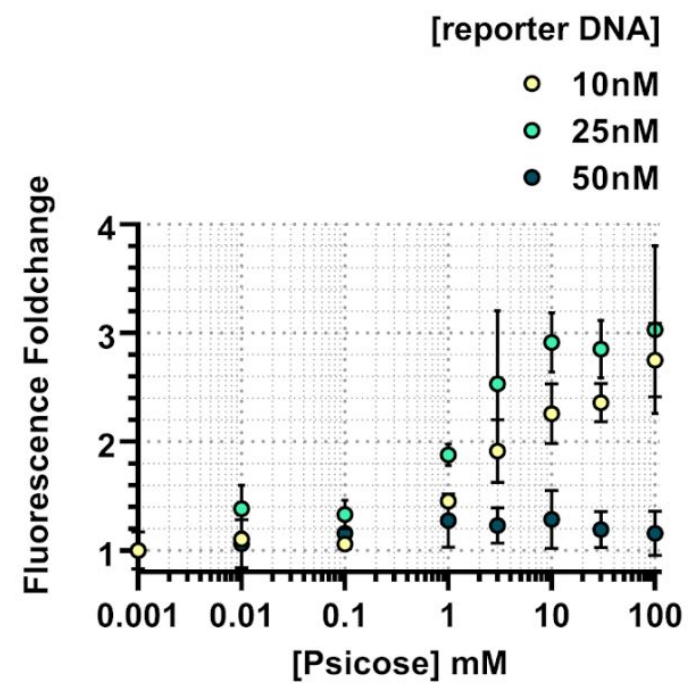

Supplementary Figure S6. Dose-response curves for $1.5 \mathrm{~h}$ preincubation experiments. Two close fold changes of $1.5 \mathrm{~h}$ preincubation from Figure 1.d. The linearity/piscose dynamic range of $10 \mathrm{nM}$ reporter DNA is higher than $25 \mathrm{nM}$ DNA. The data and error bars are the mean and standard deviation of three measurements from three independent reactions done in the same day using the same lysate and maxiprepped plasmids. 
Supplementary Table S1. List of sequences used in this study.

\begin{tabular}{|c|c|c|}
\hline Sequence name & Nucleotide sequence & $\begin{array}{l}\text { Description/ } \\
\text { Source }\end{array}$ \\
\hline $\begin{array}{l}\text { PsiR (D-psicose } \\
\text { transcription } \\
\text { factor) }\end{array}$ & $\begin{array}{l}\text { ATGACCGGTATCTCTTCTAAAAAAGCTACCATCTACGACCTGTCTA } \\
\text { TCCTGTCTGGTGCTTCTGCTTCTACCGTTTCTGCTGTTCTGAACGG } \\
\text { TTCTTGGCGTAAACGTCGTATCTCTGAAGAAACCGCTGACAAAAT } \\
\text { CCTGTCTCTGGCTAAAGCTCAGCGTTACACCACCAACTTACAGGC } \\
\text { TCGTGGTCTGCGTTCTTCTAAATCTGGTCTGGTTGGTCTGCTGGTT } \\
\text { CCGGTTTACGACAACCGTTTCTTCTCTTCTATGGCTCAGACCTTCG } \\
\text { AAGGTCAGGCTCGTAAACGTGGTCTGTCTCCGATGGTTGTTTCTG } \\
\text { GTCGTCGTGACCCGGAAGAAGAACGTCGTACCGTTGAAACCCTG } \\
\text { ATCGCTTACTCTATCGACGCTCTGTTCATCGCTGGTGTTACCGACC } \\
\text { CGGACGGTGTTCACCAGGTTTGCGCTCGTGCTGCTCTGCCGCACG } \\
\text { TTAACATCGACCTGCCGGGTAAATTCGCTTCTTCTGTTATCTCTAA } \\
\text { CAACCGTCACGGTGCTGAAATCCTGACCGCTGCTATCCTGGCTCA } \\
\text { CGCTGCTAAAGGTGGTTCTCTGGGTCCGGACGACGTTATCCTGTT } \\
\text { CGGTGGTCACGACGACCACGCTTCTCGTGAACGTATCGACGGTTT } \\
\text { CCACGCTGCTAAAGCTGACTACTTCGGTGTTGAAGGTGGTGACG } \\
\text { ACATCGAAATCACCGGTTACTCTCCGCACATGACCGAAATGGCTT } \\
\text { TCGAACGTTTCTTCGGTCGTCGTGGTCGTCTGCCGCGTTGCTTCTT } \\
\text { CGTTAACTCTTCTATCAACTTCGAAGGTCTGCTGCGTTTCATGGGT } \\
\text { CGTCACGACGGTGAAGCTTTCGGTGACATCGTTGTTGGTTGCTTC } \\
\text { GACTACGACCCGTTCGCTTCTTTCCTGCCGTTCCCGGTTTACATGA } \\
\text { TCAAACCGGACATCGCTCAGATGCTGGAAAAAGGTTTCGAACTG } \\
\text { CTGGAAGAAAACCGTACCGAACCGGAAGTTACCATCATCGAACC } \\
\text { GCAGCTGATCCCGCCGCGTACCGCTCTGGAAGGTCCGCTGGACG } \\
\text { ACATCTGGGACCCGGTTGCTCTGCGTCGTATGGCTAAATAA }\end{array}$ & $\begin{array}{l}\text { Agrobacterium } \\
\text { tumefaciens }^{1}\end{array}$ \\
\hline
\end{tabular}




\begin{tabular}{|c|c|c|}
\hline $\begin{array}{l}\text { DPEase (D-psicose } \\
\text { 3-epimerase) }\end{array}$ & $\begin{array}{l}\text { ATGAAACACGGTATCTACTACGCTTACTGGGAACAGGAATGGGA } \\
\text { AGCTGACTACAAATACTACATCGAAAAAGTTGCTAAACTGGGTTT } \\
\text { CGACATCCTGGAAATCGCTGCTTCTCCGCTGCCGTTCTACTCTGAC } \\
\text { ATCCAGATCAACGAACTGAAAGCTTGCGCTCACGGTAACGGTATC } \\
\text { ACCCTGACCGTTGGTCACGGTCCGTCTGCTGAACAGAACCTGTCT } \\
\text { TCTCCGGACCCGGACATCCGTAAAAACGCTAAAGCTTTCTACACC } \\
\text { GACCTGCTGAAACGTCTGTACAAACTGGACGTTCACCTGATCGGT } \\
\text { GGTGCTCTGTACTCTTACTGGCCGATCGACTACACCAAAACCATC } \\
\text { GACAAAAAAGGTGACTGGGAACGTTCTGTTGAATCTGTTCGTGA } \\
\text { AGTTGCTAAAGTTGCTGAAGCTTGCGGTGTTGACTTCTGCCTGGA } \\
\text { AGTTCTGAACCGTTTCGAAAACTACCTGATCAACACCGCTCAGGA } \\
\text { AGGTGTTGACTTCGTTAAACAGGTTGACCACAACAACGTTAAAGT } \\
\text { TATGCTGGACACCTTCCACATGAACATCGAAGAAGACTCTATCGG } \\
\text { TGGTGCTATCCGTACCGCTGGTTCTTACCTGGGTCACCTGCACAC } \\
\text { CGGTGAATGCAACCGTAAAGTTCCGGGTCGTGGTCGTATCCCGT } \\
\text { GGGTTGAAATCGGTGAAGCTCTGGCTGACATCGGTTACAACGGT } \\
\text { TCTGTTGTTATGGAACCGTTCGTTCGTATGGGTGGTACCGTTGGT } \\
\text { TCTAACATCAAAGTTTGGCGTGACATCTCTAACGGTGCTGACGAA } \\
\text { AAAATGCTGGACCGTGAAGCTCAGGCTGCTCTGGACTTCTCTCGT } \\
\text { TACGTTCTGGAATGCCACAAACACTCTTAA }\end{array}$ & $\begin{array}{l}\text { Clostridium } \\
\text { cellulolyticum }{ }^{1}\end{array}$ \\
\hline $\begin{array}{l}\text { pPsiA (responsive } \\
\text { promoter to psiR) }\end{array}$ & $\begin{array}{l}\text { GTATAAATGGTGGCTTTTTTTGAACTTATGCCCGTCACTGTGATCT } \\
\text { CCCCAACTGATTCCGATTATTAGAGCACGCATCCCCTTGACGGAA } \\
\text { GGGCGCTTCATGATATGGTTATTGCACCATCGATTGTGCAGATTG } \\
\text { GCAATATCGATTGTGCATGGTGGTTGCTATGGGAGTGGCAAGGG } \\
\text { AGAGTCTCGAATAAGCGAGATGAGAGATTTTGAACGCGTCCGGG } \\
\text { AAAAACGGGCTGCGGGCGGATTTCGTTTGCCGAATTTTTGAGGA } \\
\text { GGAACATCAATGAAGAAAATTATTGCTGCGGCGGTTGGTCTGTC } \\
\text { GCTGGCGTTGCTCTCATCCGCAGCCTTTGCCGAAGGGCCGAAGGT } \\
\text { GGGCGTCGTCGTCAAGATCGGCGGCATTCCGTGGTTCAACGCCA } \\
\text { GCAGCCATGGGTACAAATGGAGGAAAAGAGGAGAAAAGATCAA } \\
\text { TG }\end{array}$ & $\begin{array}{l}\text { Agrobacterium } \\
\text { tumefaciens }{ }^{1}\end{array}$ \\
\hline
\end{tabular}




\begin{tabular}{|c|c|c|}
\hline sfGFP & $\begin{array}{l}\text { ATGCGTAAAGGCGAAGAGCTGTTCACTGGTGTCGTCCCTATTCTG } \\
\text { GTGGAACTGGATGGTGATGTCAACGGTCATAAGTTTTCCGTGCGT } \\
\text { GGCGAGGGTGAAGGTGACGCAACTAATGGTAAACTGACGCTGA } \\
\text { AGTTCATCTGTACTACTGGTAAACTGCCGGTACCTTGGCCGACTCT } \\
\text { GGTAACGACGCTGACTTATGGTGTTCAGTGCTTTGCTCGTTATCC } \\
\text { GGACCATATGAAGCAGCATGACTTCTTCAAGTCCGCCATGCCGGA } \\
\text { AGGCTATGTGCAGGAACGCACGATTTCCTTTAAGGATGACGGCA } \\
\text { CGTACAAAACGCGTGCGGAAGTGAAATTTGAAGGCGATACCCTG } \\
\text { GTAAACCGCATTGAGCTGAAAGGCATTGACTTTAAAGAAGACGG } \\
\text { CAATATCCTGGGCCATAAGCTGGAATACAATTTTAACAGCCACAA } \\
\text { TGTTTACATCACCGCCGATAAACAAAAAAATGGCATTAAAGCGAA } \\
\text { TTTTAAAATTCGCCACAACGTGGAGGATGGCAGCGTGCAGCTGG } \\
\text { CTGATCACTACCAGCAAAACACTCCAATCGGTGATGGTCCTGTTC } \\
\text { TGCTGCCAGACAATCACTATCTGAGCACGCAAAGCGTTCTGTCTA } \\
\text { AAGATCCGAACGAGAAACGCGATCATATGGTTCTGCTGGAGTTC } \\
\text { GTAACCGCAGCGGGCATCACGCATGGTATGGATGAACTGTACAA } \\
\text { ATGATGATAA }\end{array}$ & $\begin{array}{l}\text { Super folder } \\
\text { GFP }\end{array}$ \\
\hline $\begin{array}{l}\text { J23101 } \\
\text { constitutive } \\
\text { promoter + B0032 } \\
\text { RBS (expressing } \\
\text { the TF and } \\
\text { enzyme genes) }\end{array}$ & $\begin{array}{l}\text { AGGATACTAGAGGATGACCCCATCTGTTTACAGCTAGCTCAGTCC } \\
\text { TAGGTATTATGCTAGCTAGTAGAGTCACACAGGAAAGTAGTAGA } \\
\text { TG }\end{array}$ & iGEM registry 2 \\
\hline
\end{tabular}




\section{Supplementary References:}

(1) http://2017.igem.org/Team:Evry_Paris-Saclay/Part

(2) parts.igem.org. 